\title{
FATORES ASSOCIADOS AO CONSUMO ENERGÉTICO DE ADOLESCENTES BRASILEIROS DO ESTUDO BRASILEIRO DE NUTRIÇÃO E SAÚDE (EBANS) \\ Pôster
}

Autores deste trabalho:

Mauro Fisberg: - Instituto Pensi, Fundação Jose Luiz Egydio Setubal, Sabará Hospital Infantil, São Paulo, Brasil -Departamento de Pediatria, Escola Paulista de Medicina, Universidade Federal de São Paulo, São Paulo, Brasil

Aline Veroneze de Mello: Departamento de Nutrição, Faculdade de Saúde Pública, Universidade de São Paulo, São Paulo, Brasil

Ágatha Nogueira Previdelli: Faculdade de Ciências Biológicas e da Saúde, Universidade São Judas Tadeu, São Paulo, Brasil

Gerson Luis de Moraes Ferrari: - Centro de Investigación en Fisiologia del Ejercicio (CIFE), Universidad Mayor, Santiago, Chile - Disciplina de Alergia, Imunologia Clínica e Reumatologia do Departamento de Pediatria da Universidade Federal de São Paulo (UNIFESP), São Paulo, Brasil

Natasha Aparecida Grande de França: Departamento de Nutrição, Faculdade de Saúde Pública, Universidade de São Paulo, São Paulo, Brasil

Irina Kovalskys: Committee of Nutrition and Wellbeing, International Life Science Institute (ILSI-Argentina), Buenos Aires, Argentina

Área do Trabalho: Nutrição

Data da submissão: 30/07/2018 às 12:33

\section{Justificativa}

A adolescência, fase de transição para vida adulta, vem sendo caracterizada por uma série de modificações nos quais se encontram as alterações na composição corporal e nos hábitos alimentares. Tais mudanças têm impactado no surgimento de obesidade e outras doenças associadas, sendo essencial o monitoramento do consumo energético para a prevenção das mesmas.

\section{Objetivo(s)}

Investigar os fatores associados ao consumo energético em adolescentes brasileiros.

\section{Método(s)}

Foram utilizados dados de adolescentes do Estudo Brasileiro de Nutrição e Saúde (EBANS), parte do estudo ELANS (Estudo Latino-Americano de Nutrição e Saúde). Trata-se de um estudo transversal, com amostra representativa de adolescentes (15 a 19 anos; $n=235)$, residentes em áreas urbanas brasileiras. O consumo energético (em kcal) foi obtido por meio de recordatórios alimentares de $24 \mathrm{~h}$ (R24h) e os fatores associados ao consumo energético foram estimados por modelo de regressão linear múltiplo ajustado por sub/superrelato e nível de atividade física. As análises foram realizadas no software STATA 13.0. O estudo foi aprovado pelo Comitê de Ética em Pesquisa (31670314.8.0000.5567).

\section{Resultado(s)}




\section{$4^{\circ}$ Congresso Internaciona! \\ Seabogaró \\ 13 a 15 de \\ 13 a 15 de 2018}

Hotel Maksoud Plaza
Alameda Campinas, 150
Säo Paulo- Brosil

Independentemente do nível de atividade física e sub/superrelato de energia, houve uma associação positiva entre o consumo energético com estado nutricional e macrorregiões brasileiras, e por outro lado, uma associação negativa entre o consumo energético com sexo feminino. Maior consumo energético médio foi identificado entre adolescentes do sexo masculino, do Centro-Oeste e com obesidade, seguido de sobrepeso.

\section{Conclusão (ões)}

Os achados apontam que os fatores sexo, macrorregiões brasileiras e estado nutricional estão associados ao consumo energético de adolescentes brasileiros. Esses resultados reforçam a importância de focar estratégias de contenção do avanço da obesidade e políticas de alimentação e nutrição em regiões específicas do país e nas faixas etárias mais jovens, considerando diferenças entre os sexos. 